\title{
Effect of a Combination of Berberine and Monacolin on CYP Enzymes: An Oral Cocktail Interaction Study in Healthy Volunteers
}

Stefano Persiani ${ }^{1}$, Federica Sala ${ }^{1}$, Carla Manzotti ${ }^{1}$, Milena Colovic ${ }^{1}$, Yves Donazzolo ${ }^{2}$, Beatrice Barbetta ${ }^{1}$, Cristina Vitalini ${ }^{1}$, Giampaolo Giacovelli ${ }^{1}$, Claudio Benvenuti ${ }^{1}$ and Lucio C. Rovati ${ }^{1}$

1. R\&D Division, Rottapharm|Madaus, Monza 20900, Italy;

2. Eurofins|Optimed, Gieres 38610, France

\begin{abstract}
Objectives: This study was aimed at investigating the potential of the herbal exctracts, berberine and monacolin, administered in combination at the recommended dose, to generate clinically relevant inhibition of the CYP (cytochromes P450) enzyme system after a single oral administration in human healthy volunteers. Methods: Twelve healthy male volunteers received a five-probe drug cocktail alone (reference) or with the combination of berberine and monacolin (test), in a randomized, open label, crossover fashion. Plasma concentrations of cocktail components, caffeine (CYP1A2), metoprolol (CYP2D6), omeprazole (CYP2C19), midazolam (CYP3A4), warfarin (CYP2C9), and plasma concentrations of berberine, monacolin and its metabolite were measured by LC-MS/MS. Pharmacokinetic parameters were determined by non-compartmental analysis. Lack of inhibition was assumed if the $90 \%$ CI (confidence interval) for estimated ratio test/reference was included in the acceptance limits $0.70-1.43$ for phenotyping metrics $\mathrm{AUC}_{\text {last }}$ and $\mathrm{C}_{\max }$. Key findings: All test/reference ratios were close to unity and CIs (confidence intervals) were within the acceptance limits, except for the upper value of omeprazole $\mathrm{C}_{\max }$. Given the high intraindividual variability, this finding was considered clinically irrelevant. Conclusions: Clinically relevant inhibition of the activities of the CYP isoenzymes investigated can be excluded when berberine and monacolin are administered in combination at the recommended dose.
\end{abstract}

Key words: Herbal extracts, cocktail, cytochrome P450, berberine, monacolin, healthy volunteers.

\section{Introduction}

Use of food supplements containing herbal extracts has risen dramatically and concomitant intake with conventional medications can be expected. This generates the potential for herb-drug interactions and represents a serious public health concern. To date, data on potential herb-drug interactions are scarce and the underlying mechanisms are not sufficiently investigated [1]. Moreover, it is difficult to predict and transfer the risk from one product to another, due to the variability in herbal product composition, extraction procedures and/or excipients [2].

The herbal products objective of the present

Corresponding author: Stefano Persiani, Ph.D., research fields: translational sciences and pharmacokinetics. E-mail: stefano.persiani@ rottapharmbiotech.com. investigation, berberine hydrochloride and fermented red yeast rice extract (Monascus purpureus) which contains monacolin, are marketed in several European countries alone, but also in combination and they are well described for their lipid-lowering properties [3-5]. Berberine, a natural plant extract from Berberis aristata bark, reduces serum cholesterol by increasing the LDL (low-density lipoprotein) receptor on the liver cell surface, and inhibits triglycerides biosynthesis via the activation of AMP activated proteokinase [5, 6]. Monacolin, also known as lovastatin lactone, lowers cholesterol by inhibiting the activity of

HMG-CoA (3-hydroxy-3-methylglutaryl-coenzyme A) reductase, the rate limiting step of hepatic cholesterol biosynthesis [7]. In clinical studies, the combination 
of red yeast rice and berberine, significantly improved the lipid profile in subjects with altered cholesterol and/or triglycerides or in subjects intolerant to statins [5]. Another study was the first documenting that this combination with dietary counseling, decreased the prevalence of metabolic syndrome and reduced the risk of coronary heart disease [8]. These data were confirmed in an extended multicenter-randomized clinical trial enrolling dyslipidemic subjects [9].

Given the target population, the marketed food supplements containing berberine and monacolin in combination could be taken concomitantly with other drugs and clinically relevant CYP inhibition are possible. Berberine is metabolised predominantly by CYP2D6, but also by CYP3A4 and CYP1A2 [10, 11] and was found to be an inhibitor of CYP2D6, CYP3A4 and CYP2C9 [12]. Monacolin is metabolized by CYP3A4 $[13,14]$ and was found to be an inhibitor of CYP2C9, CYP2C19 and CYP3A4 [15]. CYP inhibition is very often responsible for clinically relevant drug interaction and there is increasing evidence that herb-drug interactions may be attributable to the inhibition of hepatic and intestinal drug-metabolizing enzymes by herbs [2]. Notwithstanding that there are few and incomplete studies on metabolic inhibition interactions with herbal extracts as perpetrators. It is therefore important to assess the potential inhibition of the marketed combination of berberine and monacolin as perpetrator to predict and/or exclude clinically relevant inhibition of the activities of the major human CYP isoenzymes.

Since regulatory guidances $[16,17]$ suggest clinical trials (rather than in vitro studies) and a validated cocktail approach as a way to evaluate potential metabolic inhibition-based drug interactions, the present study used an oral cocktail of five probe drugs that was previously validated [18]. Each drug is a specific substrate of the major isoforms of CYP: caffeine for CYP1A2, metoprolol for CYP2D6, omeprazole for CYP2C19, midazolam for CYP3A4 and warfarin for CYP2C9. The present study investigated if a marketed combination of berberine and monacolin administered at the single recommended dose inhibits the activity of these CYP enzymes in man. For this aim, the bioavailability of the five probe drugs in healthy male volunteers when the five-probe drug cocktail was administered alone (reference treatment) or with the combination of berberine and monacolin (test treatment), was determined.

\section{Materials and Methods}

\subsection{Materials}

In order to study a marketed combination of berberine and monacolin that is fully characterized for its content (active ingredients and excipients), and for which safety and efficacy data are available from clinical trials aimed at evaluating its lipid-lowering activity, the food supplement ArmoLIPID PLUS, obtained from Rottapharm|Madaus (Monza, Italy), was used in the present investigation. The five probe drugs of the cocktail were provided by Eurofins Optimed (Gieres, France). The analytical standards berberine, lovastatin lactone, caffeine, metoprolol, omeprazole, midazolam and warfarin were purchased from Sigma-Aldrich (St. Louis, MO, USA) and lovastatin hydroxy acid from Cayman Chemical (Ann Arbor, MI, USA). Solvents were obtained from standard sources and were of HPLC grade.

\subsection{Clinical Study Design}

A single centre, open label, randomized, crossover and single oral dose study was conducted in twelve healthy male volunteers who received the validated five-probe drug cocktail alone (reference treatment) and the five-probe drug cocktail concomitantly with the combination of berberine and monacolin (test treatment) with at least 15 days washout between each period. The volunteers were hospitalized for each of the periods until INR (international normalized ratio) returned to baseline value $\pm 10 \%$ or INR $<2$.

The study was carried out at Eurofins Optimed 
(Gieres, France) in accordance with the Declaration of Helsinki as modified in Seoul (2008), with the recommendations on good cinical practice (ICH E6) and with any applicable local regulatory requirements. The study was approved by the local Ethics Committee "Comitè de Protection des Personnes Sud Est IV" (CPP n : 13/022, deliberation ref: A 13-276, 24 July 2013) and by the French Health Authorities "ANSM", Agence Nationale de Sécurité du Medicament et des produits de santé (130434A-21, 4 June 2013). All subjects provided written informed consent.

The participants had to be in good health condition according to medical history, physical examination, ECG (electrocardiogram), and clinical laboratory measurements. The subjects were not allowed to smoke more than five cigarettes a day.

Intake of food and beverages was standardized and consumption of the following was prohibited from 12 days prior to each dosing: St John Worth, echinacea, ginko biloba, ginseng, Co-enzime Q10, goldenseal, garlic and products containing iron, and from 7 days for cruciferus vegetables. Grapefruit or orange juice, as well as excessive consumption of beverages containing xanthine, were to be avoided 5 days prior to each dosing and 3 days before the beginning of the study, respectively. Moreover, within 3 months prior to the screening day, any medications or nutraceuticals intake that may interfere with the pharmacokinetics of the test compounds were prohibited.

\subsection{Drug, Dose and Plasma Sampling}

During the reference treatment phase, the five-probe drug cocktail was administered on Day 1 as described [18] with $240 \mathrm{~mL}$ of tap water. The cocktail contained $100 \mathrm{mg}$ caffeine, $100 \mathrm{mg}$ metoprolol, $20 \mathrm{mg}$ omeprazole, $0.03 \mathrm{mg} / \mathrm{Kg}$ midazolam and $10 \mathrm{mg}$ warfarin. During the test treatment, the cocktail was administered on Day 1 together with one tablet (the recommended dose) of the combination containing $500 \mathrm{mg}$ of berberine, $200 \mathrm{mg}$ of red yeast rice extract
(Monascus purpureus) equivalent to $3 \mathrm{mg}$ of monacolin, $10 \mathrm{mg}$ of policosanol, $0.2 \mathrm{mg}$ folic acid, $2.0 \mathrm{mg}$ coenzyme Q10 and $0.5 \mathrm{mg}$ astaxanthin (ArmoLIPID PLUS, Rottapharm|Madaus, Monza, Italy). All treatments were administered in sitting position and in fasting conditions that were maintained for $4 \mathrm{~h}$ after drug intake.

The sampling times for the cocktail components determinations in plasma were: pre dose, $0.5,1,1.5,2$, $3,4,6,8,12,16,24$ and $48 \mathrm{~h}$. Additional samples were collected for warfarin, every $24 \mathrm{~h}$ up to $336 \mathrm{~h}$.

When the combination was administered, blood samples were collected for the determination of berberine, monacolin and its metabolite (lovastatin hydroxy acid) at $2 \mathrm{~h}$ and $4 \mathrm{~h}$ post dosing, to ensure compliance and exposure of the subjects to the herbal extracts. Samples were stored at $-80^{\circ} \mathrm{C}$, pending analysis.

\subsection{Bioanalytical Methods}

Three different LC-MS/MS bioanalytical methods were developed at Eurofins ADME Bioanalyses (Vergeze, France) to determine the plasma concentrations of: caffeine, metoprolol, omeprazole, midazolam and warfarin.

Caffeine, metoprolol and omeprazole plasma concentrations were determined in a single assay, whereas midazolam and warfarin plasma concentrations were determined in two separate assays.

Berberine, monacolin and its metabolite plasma concentrations were determined using previously qualified LC-MS/MS bioanalytical methods [19].

Caffeine, metoprolol and omeprazole plasma samples were spiked with the following internal standards, respectively: caffeine-trimethyl- ${ }^{13} \mathrm{C}_{3}$, metoprolol- $\mathrm{d}_{7}$ and lansoprazole. Their extraction from human plasma was obtained using Oasis HLB SPE cartridges and, after evaporation under nitrogen, the reconstituted residues were analysed by triple quadrupole mass spectrometer (API 4000, AB Sciex, Framingham, MA) coupled with a HPLC system (Shimadzu Corporation, Kyoto, Japan). 
Chromatographic separation of the analytes was accomplished using a column Onyx C18 $100 \times 3 \mathrm{~mm}$ (Phenomenex, Torrance, CA) with $0.1 \%$ formic acid in water and acetonitrile as mobile phases. The mass spectrometer was operated in positive ion mode and the monitored transitions for caffeine, metoprolol and omeprazole were $195.0>138.0,268.1>116.0$ and $346.1>198.0 \mathrm{~m} / \mathrm{z}$, respectively.

Midazolam plasma samples were spiked with hydroxyalprazolam as internal standard. The sample preparation procedure was a liquid-liquid extraction with tert-butyl methyl ether. After evaporation, the reconstituted samples were injected in a HPLC-MS/MS System (Shimadzu and AB Sciex instrumentation). The compounds were separated on Kromasil 100-5 C18 (Sigma Aldrich, St. Louis, MO) and the mass spectrometer was set to monitor midazolam in the positive ion mode $(326.0>291.2 \mathrm{~m} / \mathrm{z})$.

$\mathrm{R}$ - and S-warfarin plasma samples were spiked with the internal standard ${ }^{2} \mathrm{H}_{6}$-warfarin, acidified and processed by a liquid-liquid extraction with hexane. After evaporation, the residues were reconstituted with acetonitrile. Chromatographic separation of the analytes was obtained on Astec Cyclobond I2000 $250 \times$ $4.6 \mathrm{~mm} 5 \mu \mathrm{m}$ column (Sigma Aldrich, St. Louis, MO) and using acetonitrile: acetic acid: triethylamine (1000:3:2.5, v/v/v) as mobile phase. The HPLC-MS/MS analysis was performed on a Shimadzu System coupled with an AB Sciex API4000 triple quadrupole mass spectrometer, operating in negative ion mode to monitor warfarin $(307.2>160.9$ $m / z)$.

All the developed methods were qualified by the determination of linearity on three different runs, the inter- and intra-run precision and accuracy for four concentration levels, and by the evaluation of methods' specificity.

\subsection{Pharmacokinetic Analysis}

Pharmacokinetic parameters of cocktail components were determined by non-compartmental analysis using
Kinetica version 4.3 (Thermo Electron Corporation, Philadelphia, USA). The maximum plasma concentration $\left(C_{\max }\right)$ and the corresponding time $\left(t_{\max }\right)$ were obtained directly from the plasma concentration-time data; the area under the plasma concentration-time curve from time 0 to the last measurable time point $\left(\mathrm{AUC}_{\text {last }}\right)$ was calculated according to the linear trapezoidal rule and the elimination half-life $\left(t_{1 / 2}\right)$ was calculated by the equation $t_{1 / 2}=0.693 / \mathrm{K}_{\mathrm{e}}$, where $K_{e}$ is the slope of the regression line of the terminal phase of the plasma concentration-time curve.

\subsection{Sample Size Determination and Statistical Analysis}

Intraindividual $\mathrm{CV}_{\mathrm{s}}$ were assumed not to exceed $25 \%$ for $C_{\max }$ and $\mathrm{AUC}_{\text {last, }}$ allowing the assessment of the effect of influencing factor with a sample size of 12 [20]. Log-transformed phenotyping metrics $C_{\max }$ and $\mathrm{AUC}_{\text {last }}$ were analyzed using PROC MIXED (SAS statistical package, SAS institute-Version 9.3) with treatment, sequence, period and subject-within sequence as fixed terms. Estimates and 90\% CIs (confidence intervals) for the ratio test (combination + cocktail)/reference (cocktail) were obtained by computing estimates and $90 \%$ CIs for the differences between treatment means, and then converting to ratios by the antilog transformation. For each phenotyping metric, lack of inhibition was assumed if the $90 \%$ CI for the ratio was entirely included in the acceptance range 0.70-1.43.

\section{Results}

This study used simple LC-MS/MS methods and for all analytes no interference peak were detected. The ranges, precision and accuracy values for the calibration standards are presented in Table 1 . The intra- and inter- precision and accuracy values for QC samples were within the acceptable limits, and all plasma samples were analyzed within the validated stability period. 
Table 1 Ranges of inter-assay precision and accuracy values for each compound calibration standards.

\begin{tabular}{llll}
\hline Drug & Calibration range $(\mathrm{ng} / \mathrm{mL})$ & Inter-assay precision $(\mathrm{CV} \%)$ & Accuracy $(\%)$ \\
\hline Caffeine & $25-12,500$ & $2.47-6.90$ & $-4.40-5.02$ \\
Metoprolol & $5-2,500$ & $3.97-6.16$ & $-4.85-5.28$ \\
Omeprazole & $5-2,500$ & $5.51-11.21$ & $-6.72-9.67$ \\
Midazolam & $0.1-100$ & $6.86-13.53$ & $-4.86-3.60$ \\
S-warfarin & $1-600$ & $3.54-9.00$ & $-1.47-1.47$ \\
R-warfarin & $1-600$ & $3.01-7.47$ & $-1.60-1.47$ \\
\hline
\end{tabular}
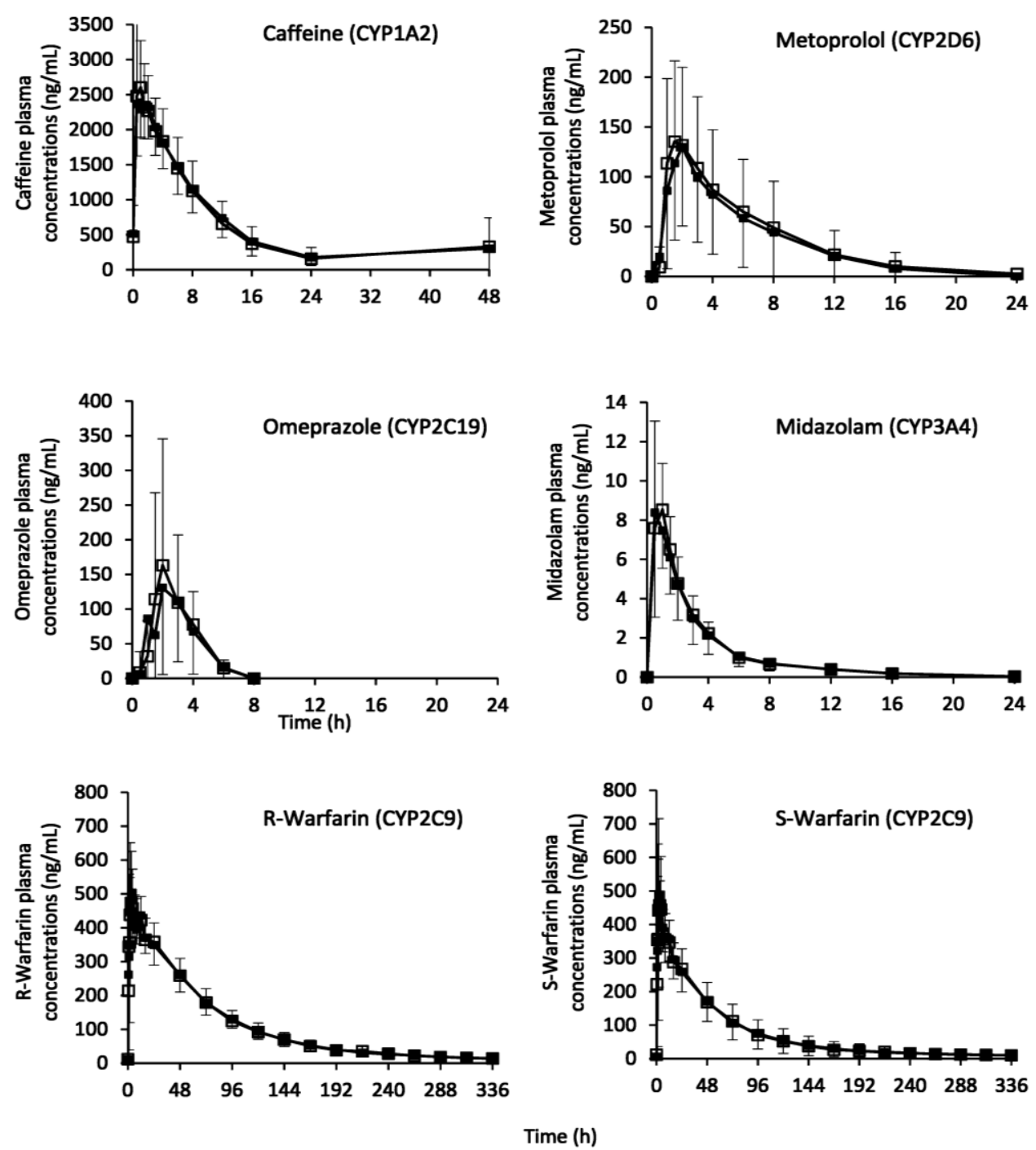

Fig. 1 Mean plasma concentrations for each probe administered as cocktail alone (reference treatment, open symbols) or with the combination of berberine and monacolin (test treatment, close symbol).

$N=12$, SD are shown by positive vertical bar for the reference treatment and by negative vertical bar for the test treatment.

Twelve healthy male subjects (11 Caucasians and 1 Black) completed the study and were included in the analysis. Their mean age was 27 years (range 20-43 years), and their mean body mass index was 22.5 (range 19.2-26.3) $\mathrm{kg} / \mathrm{m}^{2}$. At the time of the study, nine subjects were nonsmokers and three were smokers, with an average of three cigarettes/day consumption.

Mean concentration-time profiles of caffeine, metoprolol, omeprazole, midazolam, $\mathrm{R}-$ and S-warfarin when administered as drug cocktail alone 
Table 2 Summary of PK parameters for each probe drug administered during reference (cocktail alone) and test (combination + cocktail) treatment.

\begin{tabular}{lllllll}
\hline Drug & Treatment & $n$ & $C_{\max }(\mathrm{ng} / \mathrm{mL})$ & $t_{\max }(\mathrm{h})^{*}$ & $\mathrm{AUC}_{\text {last }}(\mathrm{ng} / \mathrm{mL} \cdot \mathrm{h})$ & $t_{l / 2}(\mathrm{~h})$ \\
\hline \multirow{2}{*}{ Caffeine } & Cocktail alone & 12 & $2,949(834.8)$ & $0.75(0.5-1.5)$ & $26,791(11,928)$ & $7.4(4.1)^{\mathrm{a}}$ \\
& Combination + cocktail & 12 & $2,624(560.8)$ & $1.0(0.5-2.0)$ & $27,831(11,495)$ & $5.7(2.3)^{\mathrm{b}}$ \\
\multirow{2}{*}{ Metoprolol } & Cocktail alone & 12 & $160.3(89.3)$ & $1.5(1.0-3.0)$ & $882(702)$ & $3.5(1.0)$ \\
& Combination + cocktail & 12 & $140.8(80.5)$ & $1.75(1.0-3.0)$ & $788(704)$ & $3.5(0.9)$ \\
Omeprazole & Cocktail alone & 12 & $221.3(163.4)$ & $2.0(1.0-6.0)$ & $429(277)$ & $0.9(0.1)^{\mathrm{c}}$ \\
& Combination + cocktail & 12 & $225.8(132.6)$ & $2.5(1.0-4.0)$ & $408(266)$ & $0.9(0.1)^{\mathrm{c}}$ \\
\multirow{2}{*}{ Midazolam } & Cocktail alone & 12 & $10.4(3.6)$ & $1.0(0.5-1.0)$ & $27.7(7.1)$ & $4.3(1.4)$ \\
& Combination + cocktail & 12 & $9.9(4.2)$ & $0.5(0.5-2.0)$ & $27.1(10.3)$ & $3.7(1.2)$ \\
\multirow{3}{*}{ S-warfarin } & Cocktail alone & 12 & $543.7(153.7)$ & $2.0(1.0-6.0)$ & $24,843(9,609)$ & $120.9(99.8)$ \\
& Combination + cocktail & 12 & $621.2(227.1)$ & $2.0(0.5-4.0)$ & $25,119(9,352)$ & $91.5(28.2)$ \\
R-warfarin & Cocktail alone & 12 & $537.9(131.3)$ & $2.0(1.0-6.0)$ & $36,474(7,204)$ & $83.6(37.4)$ \\
& Combination + cocktail & 12 & $576.9(182.7)$ & $3.0(0.5-12.0)$ & $36,301(5,656)$ & $67.2(14.9)$ \\
\hline
\end{tabular}

*Tabulated values are mean (SD) except for $t_{\max }$ where values are median (min, max).

${ }^{\mathrm{a}} n=7,{ }^{\mathrm{b}} n=6,{ }^{\mathrm{c}} n=5$.

Table 3 PK parameter point estimates $(90 \%$ CI) for each probe drug.

\begin{tabular}{lllll}
\hline Drug & Parameter & $n$ & Ratio estimate (90\% CI) & $\mathrm{CV}_{\text {intra }} \%$ \\
\hline \multirow{2}{*}{ Caffeine (CYP1A2) } & $\mathrm{AUC}_{\text {last }}$ & 12 & $1.04(0.86,1.27)$ & 27.1 \\
& $C_{\max }$ & 12 & $0.90(0.78,1.04)$ & 19.8 \\
Metoprolol (CYP2D6) & $\mathrm{AUC}_{\text {last }}$ & 12 & $0.90(0.77,1.06)$ & 22.1 \\
& $C_{\max }$ & 12 & $0.89(0.76,1.05)$ & 3.3 \\
Omeprazole (CYP2C19) & $\mathrm{AUC}_{\text {last }}$ & 12 & $0.94(0.71,1.25)$ & 54.2 \\
& $C_{\max }$ & 12 & $1.07(0.73,1.57)$ & 30.8 \\
Midazolam (CYP3A4) & $\mathrm{AUC}$ & $0.94(0.75,1.17)$ & 35.0 \\
S-Warfarin (CYP2C9) & $C_{\max }$ & 12 & $0.94(0.73,1.21)$ & 11.6 \\
& $\mathrm{AUC}_{\text {last }}$ & 12 & $1.01(0.93,1.11)$ & 14.4 \\
R-Warfarin (CYP2C9) & $C_{\max }$ & 12 & $1.11(1.00,1.24)$ & 9.9 \\
\hline
\end{tabular}

(reference treatment) and when administered with the combination of berberine and monacolin (test treatment) are shown in Fig. 1.

Mean values for berberine, monacolin and lovastatin hydroxy acid concentrations at 2 and $4 \mathrm{~h}$ post dosing during the test treatment were in agreement with previous studies [19] and indicated that these components were bioavailable when administered with the probe drug cocktail, thus validating the study.

PK parameters of caffeine, metoprolol, omeprazole, midazolam, R- and S-warfarin calculated following administration of the test and reference treatments are presented in Table 2. For the probe drugs, mean $C_{\max }$, $\mathrm{AUC}_{\text {last }}$ and $t_{1 / 2}$, as well as median $t_{\max }$ values, calculated during the concomitant administration of the combination, were similar to those calculated when the probe drugs were administered alone as a cocktail.

A summary of the statistical analysis results is reported in Table 3. For CYP1A2, CYP2D6, CYP3A4 and CYP2C9 phenotyping metrics, the $90 \%$ CIs for the test/reference ratios were well within the predefined acceptance limits of 0.70-1.43. Only the upper limit of the $90 \% \mathrm{CI}$ for the $C_{\max }$ ratio (1.57) of CYP2C19 probe substrate omeprazole was above the threshold of 1.43, whereas the respective value for $\mathrm{AUC}_{\text {last }}$ ratio (1.25) was within the acceptance range. Point estimates for test/reference ratios of all CYPs metrics were close to unity.

The administration of the five-probe drug cocktail alone or with the combination of berberine and monacolin was well tolerated. Two TEAEs (treatment emergent adverse events) were reported by two subjects during the study: one episode of headache after the administration of the five-probe drug cocktail 
alone and one episode of nausea after the administration of the five-probe drug cocktail with the combination of berberine and monacolin. The nausea was classified as mild in severity and resolved spontaneously, whereas the headache was moderate in severity and was treated with $500 \mathrm{mg}$ of paracetamol. Both TEAEs were judged not related to the study products. Neither clinically relevant changes nor clinically significant abnormal values were detected in the laboratory evaluation, vital signs, INR and ECG parameters over time. No abnormalities were observed in physical examination at the end of the study.

\section{Discussion}

The objective of the present study represents a relevant topic in the field of food supplements containing herbal extracts where the risk for metabolic inhibition-based drug interactions is very often unknown. Since in vitro studies are deemed questionable and animals have enzyme and transporter orthologs that differ in tissue expression or substrate specificity from those in humans, best practices to assess herb inhibition of CYP system must rely on rigorously designed clinical trials [2].

According to current guidelines [16, 17], the present clinical study was therefore conducted in healthy volunteers using a cocktail approach to assess any potential inhibition of the human CYP isoforms investigated due to the combination of berberine and monacolin administered at the recommended dose. In humans, the biotransformation of xenobiotics is mostly catalyzed by the cytochrome P450 family and it is well understood that the CYP isoenzymes $3 \mathrm{~A} 4$, $2 \mathrm{C} 9,2 \mathrm{C} 19,2 \mathrm{D} 6$, and $1 \mathrm{~A} 2$ contribute to some degree to the metabolism of more than $90 \%$ of the marketed drugs [21]. The cocktail, used in this study for CYP1A2, 2D6, 2C19, 3A4 and 2C9 enzyme activities, was previously validated [18] and can be considered suitable to investigate the potential for the combination of berberine and monacolin to inhibit the activity of the major CYP enzymes present in the human liver and involved in the metabolism of the majority of small-molecule drugs on the market. In particular for CYP3A4, the presence of midazolam in the cocktail allowed to investigate the effects of the combination on both hepatic and intestinal enzyme activities [16, 22].

In agreement with the current guideline on drug interactions [16], the main phenotyping metrics to quantify the magnitude of the mean effect of the combination berberine and monacolin on each CYP enzyme activity were obtained for each probe drug from a full concentration-time profile (AUC), in order to reduce the variability that a single-point plasma concentration might generate [18]. In addition, the assessment of AUC also allows to identify poor metabolizers and thus the need for further genotyping analysis for outliers. None of the subjects enrolled in the present study appeared to be poor metabolizers for the CYP isoforms investigated.

According to the same guideline, a compound is defined as a mild enzyme inhibitor when it causes a 1.25 to 2 fold increase in the plasma AUC of the probe substrate. In our study, the maximum observed increase in plasma AUC was 1.04 for the CYP1A2 probe substrate caffeine, indicating that the combination cannot be classified as CYP inhibitor for the activity of any of the CYP enzymes investigated in the present study. Furthermore, the statistical analysis demonstrated that the $90 \%$ CIs for the ratio test (combination + cocktail)/reference (cocktail alone) of the main phenotyping metric $\mathrm{AUC}_{\text {last }}$ of all investigated probe drugs were within the predefined acceptance limits of 0.70-1.43. These boundaries have often been used as acceptance limits to exclude drug-drug and herb-drug interactions [23-25]. Similar results were obtained in the present study for the $90 \%$ CI of the ratio (test/reference) of the second phenotyping metric $C_{\max }$ of all probe drugs, with the only exception of omeprazole. For this CYP2C19 probe substrate, the upper value of the $90 \%$ CI for the $C_{\max }$ ratio was 1.57 . However, it has to be considered 
that the intraindividual CV\% of this metric (54.2\%) was the highest of all CYPs investigated, for which the CV\% ranged between 7.1 and $35.0 \%$ (Table 2). These results are in agreement with previous investigations where omeprazole was included as a probe in cocktail interaction studies with herbal extracts $[22,23]$. As suggested by these authors this high variability is responsible for the wider $90 \% \mathrm{CI}$ rather than a significant inhibition of CYP2C19. This hypothesis is also supported by the point estimate for $C_{\max }(1.07)$, which is very close to unity, and by the fact that elimination half-life of omeprazole did not change in the presence of the combination of berberine and monacolin (Table 2), indicating that this combination did not affect the clearance of omeprazole.

The sample size of 12 evaluable subjects selected a priori in the present study proved to be sufficient for the conclusion of "no inhibition" for the phenotyping metrics of all CYP enzymes, if we do not consider the upper limit of omeprazole $C_{\max }$ for the reason explained above.

A possible limitation of the present study is that the findings may be applicable only to the combination used in this study, for which the composition is well standardized and pharmacologically investigated. Current EMA guideline [16] discourages the extrapolation of results from one herbal extracts to another, as food supplements are often a combination of extracts or herbal products and their bioavailability might be affected by the extraction process, presence of other components and/or excipients that may differ from one producer to another. Studies using the components of a food supplement administered individually are also discouraged [2] and for this reason the present investigation was conducted by administering the two herbal extracts in combination at the recommended dose.

\section{Conclusions}

The present clinical study indicates that the combination of berberine and monacolin administered at the recommended dose does not produce any clinical relevant inhibition as perpetrator toward human CYP1A2, CYP2D6, CYP2C19, CYP3A4 and CYP2C9 activities. This study is one of the few in which inhibition of the CYP system activity were assessed using a marketed food supplement, in a well controlled clinical trial. Further investigations are needed to evaluate if this combination acts as an inducer of the CYP system activities and the data of the present investigation will be useful during the interpretation of the results obtained in future studies.

\section{Acknowledgements}

This study was supported by Rottapharm|Madaus, Monza, Italy.

\section{References}

[1] Zhou, S., Lim, L. Y., and Chowbay, B. 2004. "Herbal Modulation of P-Glycoprotein." Drug Metabolism Reviews 36 (1): 57-104.

[2] Brantley, S. J., Argikar, A. A., Lin, Y. S., Nagar, S., and Paine, M. F. 2014. "Herb-Drug Interactions: Challenges and Opportunities for Improved Predictions." Drug Metabolism and Disposition 42 (3): 301-17.

[3] Cicero, A. F., Rovati, L. C., and Setnikar, I. 2007. "Eulipidemic Effects of Berberine Administered Alone or in Combination with Other Natural Cholesterol-Lowering Agents. A Single-Blind Clinical Investigation." Arzneimittelforschung 57 (1): 26-30.

[4] Trimarco, B., and Benvenuti, C. 2009. "Controlled Clinical Studies on a New Dietary Supplement Active on Cholesterol and Triglycerides in CV Risk and Metabolic Syndrome Control." Atherosclerosis Supplements; abstract 950109.

[5] Ruscica, M., Gomaraschi, M., Mombelli, G., Macchi, C., Bosisio, R., Pazzucconi, F., Pavanello, C., Calabresi, L., Arnoldi, A., Sirtori, C. R., and Magni, P. 2014. "Nutraceutical Approach to Moderate Cardiometabolic Risk: Results of a Randomized, Double-Blind and Crossover Study with Armolipid Plus." Journal of Clinical Lipidology 8 (1): 61-8.

[6] Brusq, J. M., Ancellin, N., Grondin, P., Guillard, R., Martin, S., Saintillan, Y., and Issandou, M. 2006. "Inhibition of Lipid Synthesis through Activation of AMP Kinase: An Additional Mechanism for the Hypolipidemic Effects of Berberine." Journal of Lipid Research 47 (6): 1281-8. 
[7] Heber, D. Yip, I., Ashley, J. M., Elashoff, D. A., Elashoff, R. M., and Go, V. L. 1999. "Cholesterol-Lowering Effects of a Proprietary Chinese Red-Yeast-Rice Dietary Supplement." The American Journal of Clinical Nutrition 69 (2): 231-6.

[8] Izzo, R. de Simone, G., Giudice, R., Chinali, M., Trimarco, V., De Luca, N., and Trimarco, B. 2010. "Effects of Nutraceuticals on Prevalance of Metabolic Syndrome and on Calculated Framingham Risk Score in Subjects with Dyslipidemia." Journal of Hypertension 28 (7): 1482-7.

[9] Trimarco, B., Benvenuti, C., Rozza, F., Cimmino, C. S., Giudice, R., and Crispo, S. 2011. "Clinical Evidence of Efficacy of Red Yeast Rice and Berberine in a Large Controlled Study versus Diet." Mediterranean Journal of Nutrition and Metabolism 4 (2): 133-9.

[10] Guo, Y., Li, F., Ma, X., Cheng, X., Zhou, H., and Klaassen, C. D. 2011. "CYP2D Plays a Major Role in Berberine Metabolism in Liver of Mice and Humans." Xenobiotica 41 (11): 996-1005.

[11] Li, Y., Ren, G., Wang, Y. X., Kong, W. J., Yang, P., Wang, Y. M., Li, Y. H., Yi, H., Li, Z. R., Song, D. Q., and Jiang, J. D. 2011. "Bioactivities of Berberine Metabolites after Transformation through CYP450 Isoenzymes." Journal of Translational Medicine 9: 62.

[12] Guo, Y., Chen, Y., Tan, Z. R., Klaassen, C. D., and Zhou, H. H. 2011. "Repeated Administration of Berberine Inhibits Cytochromes P450 in Humans". European Journal of Clinical Pharmacology 68 (2): 213-7.

[13] Neuvonen, P. J., Niemi, M., and Backman, J. T. 2006. "Drug Interactions with Lipid-Lowering Drugs: Mechanisms and Clinical Relevance." Clinical Pharmacology and Therapeutics 80 (6): 565-81.

[14] Jacobsen, W., Kirchner, G., Hallensleben, K., Mancinelli, L., Deters, M., Hackbarth, I., Benet, L. Z., Sewing, K. F., and Christians, U. 1999. "Comparison of Cytochrome P-450 Dependent Metabolism and Drug Interactions of the 3-Hydoxy-3-Methylglutaryl-CoA Reductase Inhibitors Lovastatin and Pravastatin in the Liver." Drug Metabolism and Disposition 27(2): 173-9.

[15] Chen, C. H., Uang, Y. S., Wang, S. T., Yang, J. C., and Lin, C. J. 2012. "Interactions between Red Yeast Rice and CYP450 Enzymes/P-Glycoprotein and Its Implication for the Clinical Pharmacokinetics of Lovastatin." Evidence-Based Complementary and Alternative Medicine 2012:127043 Epub 2012 Nov 14.

[16] European Medicines Agency. 2012. "Guideline on the Investigation of Drug Interactions". Committee for Human Medicinal Products. http://www.ema.europa.eu/docs/en_GB/document_library /Scientific_guideline/2012/07/WC500129606.pdf.

[17] Food and Drug Administration. 2012. "Guidance for
Industry. Drug Interaction Studies-Study Design, Data Analysis, Implications for dosing, and Labeling Recommendations". Rockville, MD: US Department of Health and Human Services, Food and Drug Administration, Center for Drug Evaluation and Research, http://www.fda.gov/downloads/RegulatoryInformation/G uidance/UCM292362.pdf.

[18] Turpault, S., Brian, W., Van Horn, R., Santoni, A., Poitiers, F., Donazzolo, Y., and Boulenc, X. 2009. "Pharmacokinetic assessment of five-probe cocktail for CYPs 1A2, 2C9, 2C19, 2D6 and 3A". British Journal of Clinical Pharmacology 68 (6): 928-35.

[19] Persiani, S., Sala, F., Manzotti, C., Donazzolo, Y., Benvenuti, C., Rovati, L.C. 2014. "Bioavailability of Berberine and Monacolin after Oral Administration of a Proprietary Nutraceutical Combination in Healthy Male Volunteers." Atherosclerosis 235 (2): e297. EAS-1143. http://dx.doi.org/10.1016/j.atherosclerosis.2014.05.895.

[20] Fuhr, U., Jetter, A., and Kirchheiner, J. 2007. "Appropriate phenotyping procedures for drug metabolizing enzymes and transporters in humans and their simultaneous use in the "Cocktail" approach". Clinical Pharmacology and Therapeutics 81 (2): 270-83.

[21] Guengerich, F. P. 2008. "Cytochrome p450 and Chemical Toxicology". Chemical Research in Toxicology 21 (1): 70-83.

[22] Huang, S.M., Temple, R., Throckmorton, D.C., and Lesko, L.J. 2007. "Drug Interaction Studies: Study Design, Data Analysis, and Implications for Dosing and Labeling". Clinical Pharmacology and Therapeutics 81 (2): 298-304, Review.

[23] Tomalik-Scharte, D., Jetter, A., Kinzig-Schippers, M., Skott, A., Sörgel, F., Klaassen, T., Kasel, D., Harlfinger, S., Doroshyenko, O., Frank, D., Kirchheiner, J., Bräter, M., Richter, K., Gramatté, T., and Fuhr, U. 2005. "Effect of Propiverine on Cytochrome P450 Enzymes: A Cocktail Interaction Study in Healthy Volunteers." Drug Metabolism and Disposition 33 (12): 1859-66.

[24] Doroshyenko, O., Rokitta, D., Zadoyan, G., Klement, S., Schläfke, S., Dienel, A., Gramatté, T., Lück, H., and Fuhr, U. 2013. "Drug Cocktail Interaction Study on the Effect of the Orally Administered Lavender Oil Preparation Silexan on Cytochrome P450 Enzymes in Healthy Volunteers." Drug Metabolism and Disposition 41 (5): 987-93.

[25] Zadoyan, G., Rokitta, D., Klement, S., Dienel, A., Hoerr, R., Gramatté, T., and Fuhr, U. 2012. "Effect of Ginkgo Biloba Special Extract EGb 761® on Human Cytochrome P450 Activity: A Cocktail Interaction Study in Healthy Volunteers." European Journal of Clinical Pharmacology 68 (5): 553-60. 